\title{
Angular redistribution of nonlinear perturbations: A universal feature of nonuniform flows
}

\author{
W. Horton, ${ }^{1}$ J.-H. Kim, ${ }^{2}$ G. D. Chagelishvili, ${ }^{3,4}$ J. C. Bowman, ${ }^{5}$ and J. G. Lominadze ${ }^{3,4}$ \\ ${ }^{1}$ Institute for Fusion Studies, The University of Texas at Austin, Austin, Texas 78712, USA \\ ${ }^{2}$ Department of Physics, University of Wisconsin at Madison, Madison, Wisconsin 53706, USA \\ ${ }^{3}$ Georgian National Astrophysical Observatory, The Chavchavadze State University, Tbilisi 0160, Georgia \\ ${ }^{4}$ M. Nodia Institute of Geophysics, Tbilisi 0193, Georgia \\ ${ }^{5}$ Department of Mathematical and Statistical Sciences, University of Alberta, Edmonton, Alberta, Canada T6G 2 G1
}

(Received 17 November 2009; published 9 June 2010)

\begin{abstract}
Classically, the net action of nonlinear turbulent processes is interpreted as either a direct or inverse cascade. However, in nonuniform/shear flows the dominant process is a nonlinear redistribution over wave number angle of perturbation spatial Fourier harmonics. We call this process a nonlinear transverse redistribution (NTR). This phenomenon is demonstrated for a simple two-dimensional constant shear (non-normal) flow by numerically simulating the nonlinear dynamics of coherent and stochastic vortical perturbations in the flow. NTR is a general feature of nonlinear processes that should manifest itself in nonuniform engineering, environmental, and astrophysical flows. The conventional characterization of turbulence in terms of direct and inverse cascades, which ignores NTR, appears to be misleading for shear flow turbulence. We focus on the action of nonlinear processes on the spectral energy. NTR redistributes perturbations over different quadrants of the wave number plane and the interplay of this nonlinear redistribution with linear phenomena becomes intricate: it can realize either positive or negative feedback. In the case of positive feedback, it repopulates the quadrants in wave number space where the shear flow induces linear transient growth.
\end{abstract}

DOI: 10.1103/PhysRevE.81.066304

\section{INTRODUCTION}

Nonuniform flows are ubiquitous both in nature and in the laboratory: they occur in atmospheres, oceans, stars, protoplanetary disks, galaxies, pipe flows, and tokamak reactors. The understanding of nonuniform kinematics associated with dynamical phenomena is as equally important as thermodynamic inhomogeneity. Indeed, the appearance of complex dynamics is often a manifestation of nonuniform kinematics. For example, (i) the structure and dynamical appearance of astrophysical disks are largely defined by the differential character of the disk matter rotation, (ii) turbulent flow in many engineering circumstances is governed by a nonuniform velocity profile, (iii) the encouraging $\mathrm{L}-\mathrm{H}$ transition in tokamaks is connected with the appearance of a background poloidal nonuniform $\boldsymbol{E} \times \boldsymbol{B}$ flow.

Historically, the non-normality of nonuniform (shear) flows substantially delayed the full understanding of their behavior. In fact, the non-normal nature of shear flow and its consequences only became well understood by the hydrodynamic community in the 1990s [1-4]. Shortcomings of traditional modal analysis (spectral expansion of perturbations in time and, later, eigenfunction analysis) for shear flows have been revealed. Operators in the mathematical formalism of shear flow modal analysis, such as for plane Couette and Poiseuille flows, are non-normal and the corresponding eigenmodes are nonorthogonal $[1,4,5]$. The nonorthogonality leads to strong interference among the eigenmodes. Consequently, a proper approach should fully analyze eigenmode interference. While possible in principle, this is in practice a formidable task. The mathematical approach was therefore changed, and a breakthrough in the understanding and precise description of linear transient phenomena ensued. In spectrally stable hydrodynamic shear flows, it became clear that vortex and wave mode perturbations of certain spatial characteristics/orientation undergo linear transient growth
PACS number(s): 47.27.W-, 47.27.T-, 47.27.Cn

$[6,7]$, leading to short perturbation life times. However, in certain favorable circumstances, the perturbations are sustained long by the shear flows. Specifically, the imperfect linear growth must be compensated by the nonlinear positive feedback that repopulates perturbations transiently by drawing energy from the shear flow. In this case, the nonlinearity closes the feedback loop, producing self-sustaining perturbations. In the 1990s, on the basis of this interplay between linear transient growth and nonlinear positive feedback, the hydrodynamic community formulated the bypass transition concept to explain the onset of turbulence in spectrally stable shear flows (see, e.g., [8-12]). Early hypotheses of the bypass concept can be found in Refs. $[13,14]$. These papers, which examine the continuous spectrum of the OrrSommerfeld equation, primarily discuss linear processes, but nevertheless contain some speculation on the mechanisms by which the continuous spectrum could lead to a bypass transition.

The bypass scenario differs principally from the typical turbulence scenario, which is based on exponentially growing perturbations in a system that supplies turbulent energy and requires no nonlinear positive feedback to maintain the perturbations. In the classic case the nonlinearity is not vital to the existence of the perturbations; instead, it merely determines their scales, via the direct and/or inverse cascade.

The aim of this work is to describe the general behavior of nonlinear processes of the Navier-Stokes equations by direct numerical simulation. To supplement Kida's elegant kinematic description of how an elliptic vortex responds to a uniform shear flow [15], we follow the dynamics of coherent (cyclonic/anticyclonic) and stochastic vortical perturbations in a prototypical two-dimensional (2D) constant shear flow. Since our focus is on nonlinear phenomena, we do not invoke methods such as rapid distortion theory that deal primarily with linear transient dynamics. 
We also do not attempt to model turbulence. Instead, we simply highlight a novel feature of the nonlinearity in shear flows. Usually, linear processes in different systems depend on some combination of wave number values, say (in the $2 \mathrm{D}$ case) $k_{x}^{2}$ and $k_{y}^{2}$. Therefore, nonlinear processes can increase or decrease $k_{x}^{2}$ and $k_{y}^{2}$, leading to direct or inverse cascades. In our case, flow velocity shear introduces a dependency of the linear processes on the combination $k_{x} k_{y} / k^{2}$. Nonlinear processes can thus lead to changes in the sign of $k_{x} k_{y}$ due to nonlinear redistribution of perturbation Fourier harmonics over wave number angle. We call this process nonlinear transverse redistribution (NTR). It is important to stress that the nonlinear processes redistribute perturbations between different quadrants of the wave number plane: from $k_{x} k_{y}$ $>0$ to $k_{x} k_{y}<0$, or the reverse. Such nonlinear processes are impossible in the usual cases where linear processes depend only on some combination of $k_{x}^{2}$ and $k_{y}^{2}$. As a consequence of NTR, the nonlinearity produces positive or negative feedback, and the interplay of linear and nonlinear phenomena becomes intricate. The nonlinearity acquires a vital role: it enables the self-sustenance or self-suppression of regular or stochastic perturbations.

\section{BASIC EQUATIONS AND PROCESSES}

Let us illustrate NTR for localized, coherent, and stochastic perturbations in a 2D spectrally stable incompressible hydrodynamic uniform shear flow, $\boldsymbol{U}_{0}(x)=(0, A x)$, with constant mean pressure and density. The same underlying mechanism should be relevant to three-dimensional (3D) shear flows and in more complex media such as plasmas. The dynamical equation written for the stream function $\psi$ has the form

$$
\left(\frac{\partial}{\partial t}+\boldsymbol{U}_{0}(x) \cdot \boldsymbol{\nabla}\right) \Delta \psi+J(\psi, \Delta \psi)-\nu \Delta^{2} \psi=0,
$$

where $\nu$ is the kinematic viscosity and the spatial operators $J$ and $\Delta$ represent the two-dimensional Jacobian and Laplacian, respectively. Without loss of generality, we adopt $A>0$. The velocity $\boldsymbol{v}$ and energy density $e$ of the perturbation are defined in terms of the stream function and the constant fluid mass density $\rho_{0}$,

$$
\begin{gathered}
\boldsymbol{v}=\left(v_{x}, v_{y}\right)=\hat{\mathbf{z}} \times \nabla \psi=\left(-\frac{\partial \psi}{\partial y}, \frac{\partial \psi}{\partial x}\right), \\
e(x, y, t)=\frac{1}{2} \rho_{0}\left[\left(\frac{\partial \psi}{\partial x}\right)^{2}+\left(\frac{\partial \psi}{\partial y}\right)^{2}\right] .
\end{gathered}
$$

Introducing dimensionless variables normalized with the time scale $T$ and spatial scale $\ell$,

$$
\begin{gathered}
\tau \equiv \frac{t}{T}, \quad X \equiv \frac{x}{\ell}, \quad Y \equiv \frac{y}{\ell}, \quad \hat{A} \equiv A T, \quad R_{e} \equiv \frac{\ell^{2}}{\nu T}, \\
\Psi \equiv \frac{T \psi}{\ell^{2}}, \quad V_{x}=\frac{T v_{x}}{\ell}, \quad V_{y}=\frac{T v_{x}}{\ell}, \quad E \equiv \frac{2 T^{2} e}{\rho_{0} \ell^{2}},
\end{gathered}
$$

and performing Fourier analysis with respect to $X$ and $Y$,

$$
\Psi(X, Y, \tau)=\int d k_{x} d k_{y} \Psi_{k}(\tau) \exp \left(i k_{x} X+i k_{y} Y\right),
$$

one obtains a nonlinear equation for the dynamics of the spatial Fourier harmonics (SFHs) of the perturbations,

$$
\begin{gathered}
k^{2} \frac{\partial \Psi_{k}}{\partial \tau}-\hat{A} k_{y} \frac{\partial\left(k^{2} \Psi_{k}\right)}{\partial k_{x}}+R_{e}^{-1} k^{4} \Psi_{k}=\sum_{k=k^{\prime}+k^{\prime \prime}}\left(k_{x}^{\prime} k_{y}^{\prime \prime}\right. \\
\left.-k_{x}^{\prime \prime} k_{y}^{\prime}\right) k^{\prime 2} \Psi_{k^{\prime}} \Psi_{k^{\prime \prime}}, \\
E_{k}=k^{2}\left|\Psi_{k}\right|^{2}, \quad E(\tau) \equiv \int d k_{x} d k_{y} E_{k}(\tau),
\end{gathered}
$$

where $k^{2} \equiv k_{x}^{2}+k_{y}^{2}, E_{k}$ is twice the spectral energy density associated with the wave number $\left(k_{x}, k_{y}\right)$ and $E(\tau)$ is twice the total energy of the perturbations. On the RHS of Eq. (6) is the nonlinear term describing three-wave coupling. Equations (6) and (7) form the basis of our numerical study. With these equations, one can quantitatively explore the dynamics of the stream function, spectral energy density, and total perturbed energy. However, to investigate the physics of the phenomena, it is helpful to analyze the dynamical equation for the spectral energy density, which follows from Eqs. (6) and (7),

$$
\frac{\partial E_{k}}{\partial \tau}-\hat{A} \frac{2 k_{x} k_{y}}{k^{2}} E_{k}+\frac{\partial}{\partial k_{x}}\left(-\hat{A} k_{y} E_{k}\right)+R_{e}^{-1} k^{2} E_{k}=\hat{N}_{k},
$$

where

$$
\hat{N}_{\boldsymbol{k}}=\sum_{\boldsymbol{k}=\boldsymbol{k}^{\prime}+\boldsymbol{k}^{\prime \prime}}\left(k_{x}^{\prime} k_{y}^{\prime \prime}-k_{x}^{\prime \prime} k_{y}^{\prime}\right) k^{\prime 2} \times\left(\Psi_{\boldsymbol{k}}^{*} \Psi_{\boldsymbol{k}^{\prime}} \Psi_{\boldsymbol{k}^{\prime \prime}}+\Psi_{\boldsymbol{k}} \Psi_{\boldsymbol{k}^{\prime}}^{*} \Psi_{\boldsymbol{k}^{\prime \prime}}^{*}\right)
$$

is a functional of $\Psi_{k}$ describing the contribution of the nonlinear term to $\partial E_{k} / \partial \tau$.

One can see from Eq. (8) that the dynamics of $E_{k}$ is the result of the interplay of four basic phenomena:

(1) The second term on the LHS represents the energy exchange between the perturbations and the background flow. SFHs draw shear flow energy and are amplified in quadrants I and III of the wave number plane, where $k_{x} k_{y}$ $>0$, giving energy back to the flow in quadrants II and IV where $k_{x} k_{y}<0$. Note that quadrant III (IV) behaves analogously to I (II).

(2) The quantity $\left(-\hat{A} k_{y} E_{k}\right)$ in the third term on the LHS represents the flux of the spectral energy density along the $k_{x}$ axis with velocity $-\hat{A} k_{y}$. Each SFH "drifts" along the $k_{x}$ axis. The drift is caused by shearing of the perturbations due to the background shear flow and results in the time dependence of the SFH wave number: $k_{x}(\tau)=k_{x}(0)-\hat{A} k_{y} \tau$. Since $\int d k_{x} d k_{y} \partial($ $\left.-\hat{A} k_{y} E_{k}\right) / \partial k_{x}=0$, this drift does not change the total energy; instead, it transfers energy from the amplification region $\left(k_{x} k_{y}>0\right)$ to the attenuation region $\left(k_{x} k_{y}<0\right)$. Consequently, the linear drift gives rise to the transient character of the growth. 
(3) The fourth term on the LHS represents the energy dissipation by viscosity. The linear drift ultimately leads to an increase in the SFH wave number: $\left|k_{x}(\tau)\right|=\left|k_{x}(0)-\hat{A} k_{y} \tau\right|$ $\rightarrow \infty$, enhancing viscous dissipation.

(4) The nonlinear term $N_{k}$ on the RHS leads to energy exchange between different $\mathrm{SFH}$, redistributing perturbation energy in the wave number plane while leaving the total energy unchanged: $\Sigma_{k} N_{k}=0$. The term $N_{k}$, being responsible for NTR, is the key focus of our research. The property $\Sigma_{k} N_{k}=0$ is maintained and used to monitor the accuracy of the numerical solutions of Eq. (6).

\section{NUMERICAL SIMULATIONS}

The simulations are done using the dealiased Fourier pseudospectral method, with a $256 \times 256$ grid superimposed on a spatial box of size $25 \ell \times 25 \ell$, yielding a minimum wave number $k_{1}=2 \pi /(25 \ell)=0.25 \ell^{-1}$ and maximum wave number $k_{\max }=85 k_{1}$.

We computed the dynamic picture given by Eqs. (6)-(9) for the Fourier transform of a localized perturbation,

$$
\Psi(X, Y, 0)=B n(X, Y) \exp \left(-X^{2}-Y^{2}\right) .
$$

Thus, $n(X, Y)=1$ yields coherent circular anticyclonic perturbations, where the vorticity is antiparallel to the background vorticity $A$, while $n(X, Y)=-1$ corresponds to coherent cyclonic ones, where the vorticity is parallel to $A$. We also considered stochastic vortical perturbations, by taking $n(X, Y)$ to be the inverse Fourier transform of $\exp \left(-\left(k_{x}^{2}\right.\right.$ $\left.+k_{y}^{2}\right) / a^{2}+2 \pi i \xi_{k}$ ), using the Gaussian filter width $a=4.6$ and a random number $\xi_{k} \in[0,1]$ for each wave number $\boldsymbol{k}$, to produce several random vortices within the perturbed area. For the coherent vortexes $n(X, Y)= \pm 1$, the parameter $B=3$, the ratio of the vortex eddy turnover time to the shear time scale, is chosen to maximize the feedback [16]. The same factor $B$ is applied to the stochastic perturbations. We simulated the dynamic equations for cyclonic, anticyclonic, and stochastic perturbations for $0 \leq \tau \leq 5$ using different values of the Reynolds number $R_{e}$ and the amplitude $B$.

In the simulation, the time scale is the inverse of the shearing rate, $T=A^{-1}$. Therefore, the amplitude $B$ measures the ratio of the shearing time to the eddy turn-over time. The eddy rotation has an isotropizing effect, whereas the shear flow drives anisotropy.

For $R_{e}=1000$ and $B=3$, we present in Fig. 1 typical results that demonstrate key features of the total perturbation energy dynamics and, in Figs. 2-4, the corresponding spectral perturbation energy density $E(\boldsymbol{k})$ and nonlinear energy transfer $\hat{N}(\boldsymbol{k})$ in the wave number plane.

In Fig. 1, the evolution of the fluctuation energy $E$ normalized by the initial energy $E_{0}$ for the anticyclonic (solid red), cyclonic (dotted blue) and stochastic (dashed black) perturbations are presented. The energy of the anticyclonic vortex $(n=1)$ increases monotonically, while the energy of the cyclonic vortex $(n=-1)$ initially increases and then oscillates weakly. Since the linear dynamics of the perturbations are identical, the difference in the energy evolutions must be due to nonlinear phenomena. In the present case all types of

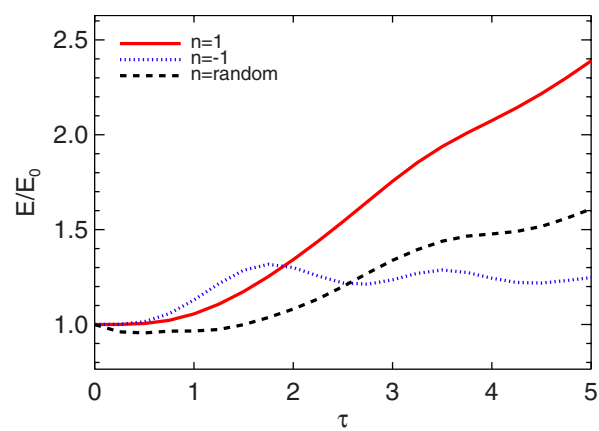

FIG. 1. (Color online) Normalized total energy vs time for anticyclonic (solid red), cyclonic (dotted blue) and stochastic (dashed black) perturbations for $R_{e}=1000$ and $B=3$

perturbations are self-sustainable during the simulation time. The energy behavior of the anticyclonic and stochastic perturbations indicates the existence of a self-consistent positive nonlinear feedback. The energy of the cyclonic perturbation oscillates in time, due to alternating positive and negative feedback. These insights are confirmed in Figs. 2-4.

The spectral energy density $E_{k}$ and the contribution of nonlinear processes to the spectral energy density dynamics $\hat{N}_{k}$ for an anticyclonic vortex is presented in Fig. 2 in the wave number plane at $\tau=0.5$ and 2 . The nonlinearity redistributes the perturbation energy from the blue areas $\left(\hat{N}_{k}\right.$ $<0)$ to the red ones $\left(\hat{N}_{k}>0\right)$, with the green regions indicating $\hat{N}_{k} \simeq 0$. As one can see in Figs. 2(c) and 2(d), the nonlinear interactions redistribute energy from quadrant II (IV), where $\hat{N}_{k}$ is predominantly negative, to quadrant I (III), (a)

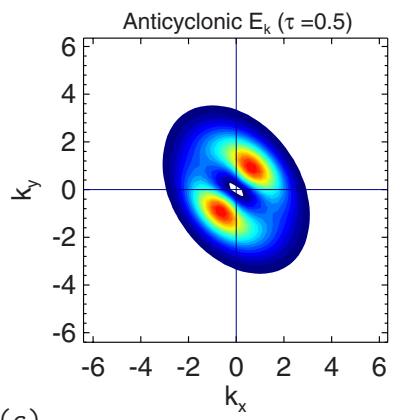

(c)

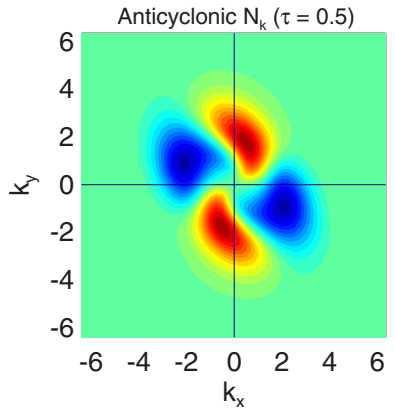

(b)

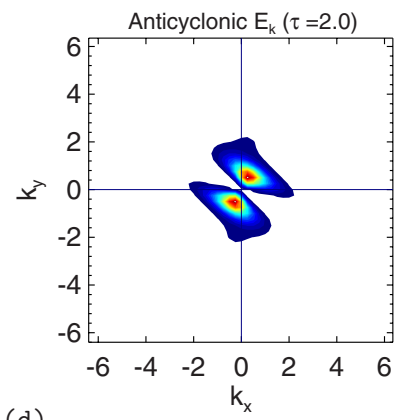

(d)

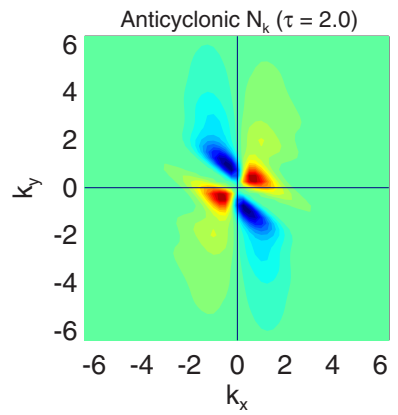

FIG. 2. (Color online) $[(\mathrm{a}),(\mathrm{b})]$ The spectral energy density $E_{k}$ [(c),(d)] the contribution $\hat{N}_{k}$ of the nonlinear processes to the spectral energy density dynamics [cf. Eqs. (8) and (9)] for an anticyclonic vortex, with $R_{e}=1000$ and $B=3$ at times $\tau=0.5$ and 2 . 
(a)

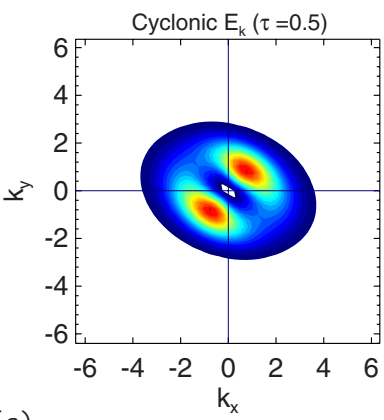

(c)

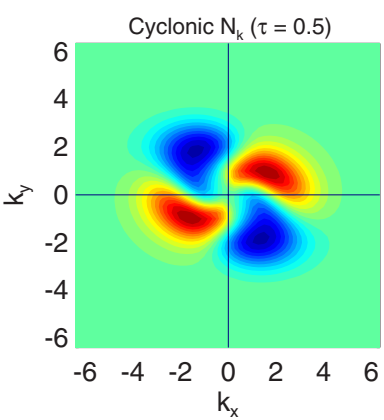

(b)

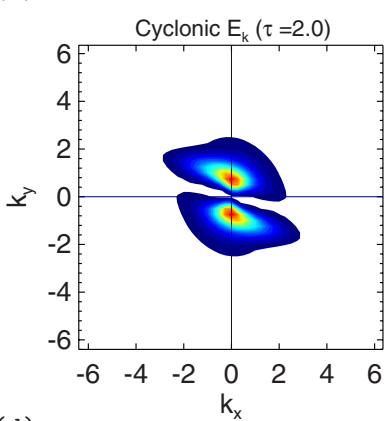

(d)

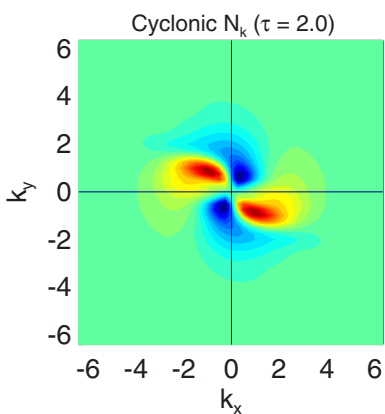

FIG. 3. (Color online) The same as in Fig. 2 but for the cyclonic vortex at times $\tau=0.5$ and 2 .

where $\hat{N}_{k}$ is predominantly positive. This angular redistribution dominates the linear drift. Therefore, the perturbation population in quadrant I (III) prevails over the perturbation population in quadrant II (IV) and $d E(\tau) / d \tau>0$ for $\tau>0$ [cf. Figs. 2(a) and 2(b)]. Indeed, the simulations for anticyclonic vortices show that nonlinear processes repopulate the growing SFHs during the simulation time $(0 \leq \tau \leq 5)$. This feedback mechanism leads to the self-sustenance of anticyclonic perturbations, so that the total energy of the anticyclonic perturbations increases, as seen in Fig. 1. Also, one can see that the domain of significant nonlinear activity converges in time to lower wave numbers: an inverse cascade is in progress. However, the dominant nonlinear process is the angular redistribution of perturbation Fourier harmonics.

Figures 3 and 4 show that NTR is also strongly pronounced for coherent cyclonic and stochastic perturbations. In the case of cyclonic vortices, the nonlinear dynamics is
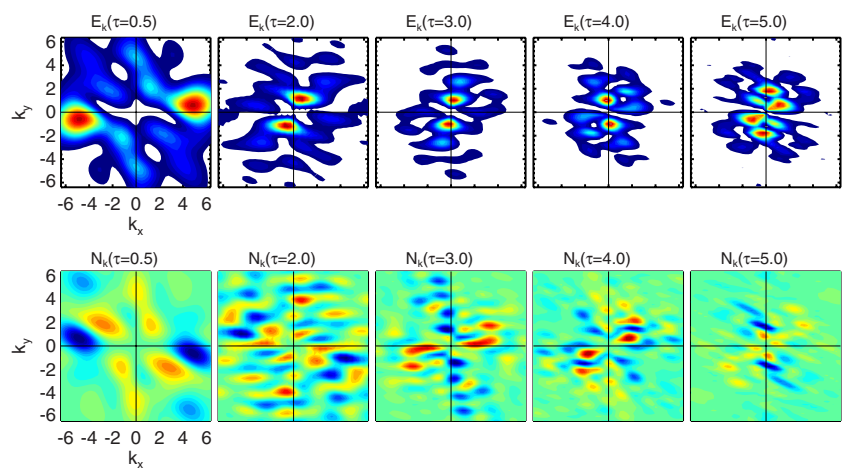

FIG. 4. (Color online) Contours of $E_{\boldsymbol{k}}$ (top) and $N_{\boldsymbol{k}}$ (bottom) are shown at $\tau=0.5,2,3,4$, and 5 . The horizontal and vertical axis represent $k_{x}$ and $k_{y}$ in the interval $[-6,6]$.

more complicated. Positive and negative feedbacks alternate in time. Indeed, in Figs. 3(c) and 3(d) we see that nonlinear interactions redistribute energy mainly in wave number angle, from quadrant II (IV) to quadrant I (III) at $\tau=0.5$ and vice versa at $\tau=2$. NTR repopulates growing SFHs for which $k_{x} k_{y}>0$ at $\tau=0.5$ [see Fig. 3(a)], contributing to an increase in the total perturbation energy. In contrast, the repopulation of decaying SFHs by the nonlinear angular redistribution [see Fig. 3(b)] leads to a decrease in the total perturbation energy at $\tau=2$ (cf. dotted blue curve in Fig. 1). So, in the case of a cyclonic vortex, the nonlinearity does not ensure a continuous positive feedback.

Figure 4 shows that the nonlinear contributions are not as smooth for the case of a stochastic perturbation. There is no well-defined positive feedback in the initial stages (see plots at $\tau=0.5$ and 2). However, at later times (see plots for $\tau=3$, 4, and 5), positive nonlinear feedback becomes pronounced, particularly at $\tau=3$. In accordance with this, $d E(\tau) / d \tau$ has a maximum in the vicinity of $\tau=3$ (cf. dashed black curve of Fig. 1). In any case, from Fig. 4 it follows that NTR and not the inverse cascade appears to be the dominant nonlinear phenomenon for the stochastic perturbations, just as in the case of coherent perturbations.

We initialized our simulations with isotropic perturbations [see Eq. (10)]. However, the spectral energy density in Figs. $2,3,4(a)$, and 4(b) indicates in each case that the perturbations became anisotropic, dominated by shearwiseindependent perturbations (i.e., ones with $k_{x}=0$ ). To characterize the nature of the transverse redistribution, the averages of $\hat{N}(\boldsymbol{k})$ introduced in the next section provide quantitative measures of NTR.

\section{QUANTITATIVE CHARACTERISTICS OF THE (DIRECT AND INVERSE) CASCADES AND NTR}

On averaging $N_{k}(\tau)$ over the polar angle $\theta$, one can characterize the intensity of the cascade with the quantity

$$
I_{\uparrow \downarrow}(k, \tau)=\frac{1}{2 \pi} \int_{0}^{2 \pi} \hat{N}_{k}(\tau) d \theta .
$$

Alternatively, on averaging over the wave number magnitude $k$, one can characterize the intensity of NTR by

$$
I_{\leftrightarrows}(\theta, \tau)=\frac{1}{k_{0}^{2}} \int_{0}^{k_{0}} \hat{N}_{\boldsymbol{k}}(\tau) k d k .
$$

The corresponding plots of the NTR intensity and direct and inverse cascades for the anticyclonic, cyclonic, and stochastic perturbations at $\tau=1.75$ are presented in Figs. 5 and 6. The NTR intensity for the stochastic perturbation at $\tau=3$, 4 , and 5 is presented in Fig. 7.

Taking $k_{0}=10$, at $\tau=1.75$ we observe in Fig. 5 the following features:

(1) Typically, for the anticyclonic perturbation, $I_{\leftrightarrows}(\theta, \tau)$ is positive for $\theta / \pi \in\left[0, \frac{1}{2}\right]$ and negative for $\theta / \pi \in\left[\frac{1}{2}, 1\right]$. This illustrates the positive feedback mechanism that redistributes SFHs from quadrant II to quadrant I and vice versa, where NTR repopulates growing SFHs for which $k_{x} k_{y}>0$. 


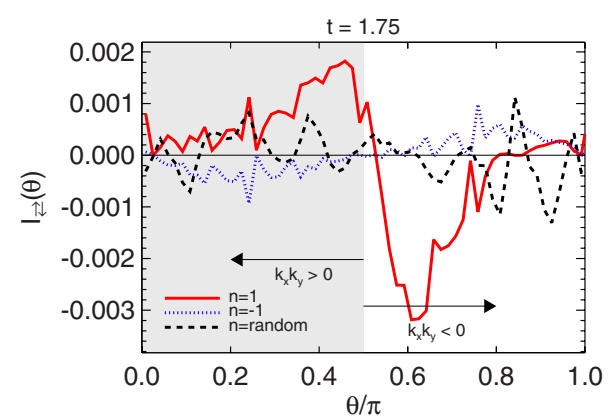

FIG. 5. (Color online) Intensity of NTR $I_{\leftrightarrows}(\theta, \tau)$ vs polar angle $\theta$ for anticyclonic (solid red), cyclonic (dotted blue), and stochastic (dashed black) perturbations at $\tau=1.75$.

(2) For the cyclonic perturbation, $I_{\leftrightarrows}(\theta, \tau)$ is negative for $\theta / \pi \in\left[0, \frac{1}{2}\right]$ and positive for $\theta / \pi \in\left[\frac{1}{2}, 1\right]$ at $\tau=1.75$. This constitutes negative feedback: NTR repopulates attenuating SFHs for which $k_{x} k_{y}<0$.

(3) For the stochastic perturbation, the repopulation is mixed and the positive feedback is not pronounced at the beginning. However, positive feedback is clearly seen in the plots of the intensities for the stochastic perturbation at $\tau$ $=3,4$, and 5 presented in Fig. 7 .

Figure 6 shows that at $\tau=1.75$ the inverse cascade is pronounced only for the anticyclonic case. The complete set of results for our numerical simulations is available from our website [17]. The results contain demonstrative movies that help one follow and comprehend the dynamical processes. Specifically, the results include movies of the dynamics of $E_{k}, \hat{N}_{k}, I_{\leftrightarrows}(\theta, \tau)$, and $I_{\uparrow \downarrow}(k, \tau)$ for anticyclonic, cyclonic, and stochastic perturbations.

\section{CONCLUSION}

In this work we demonstrated, as a first step toward understanding more realistic 3D sheared turbulent flows, the dominance of nonlinear transverse redistribution (NTR) over the direct or inverse cascades in a simple 2D shear flow. Being a general feature of nonlinear processes in shear flows, NTR should be inherent to 3D flows as well. It thus appears that the conventional characterization of turbulence solely in

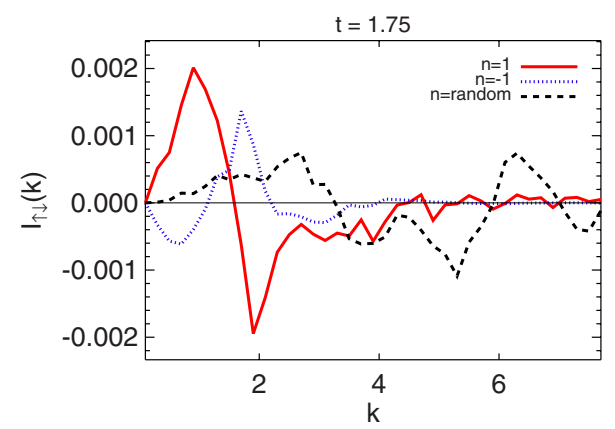

FIG. 6. (Color online) Intensity of (direct and inverse) cascades $I_{\uparrow \downarrow}(k, \tau)$ vs wave number magnitude $k$ for the anticyclonic (solid red), cyclonic (dotted blue), and stochastic (dashed black) perturbations at $\tau=1.75$.

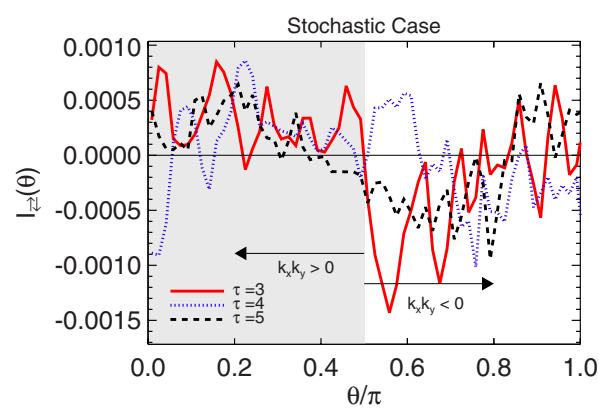

FIG. 7. (Color online) Intensity of NTR $I_{\leftrightarrows}(\theta, \tau)$ vs polar angle $\theta$ for the stochastic perturbation at $\tau=3,4$ and 5 , indicated by the solid red, dotted blue, and dashed black curves, respectively.

terms of direct and inverse cascades, which neglects NTR, is misleading for shear flow turbulence.

In our simulations, NTR ensured positive feedback and the self-sustenance of regular and stochastic perturbations. Consequently, NTR could provide a mechanism for the development of turbulent spots from stochastic perturbations, following the bypass scenario of turbulence.

According to our simulations (see the dynamics of $E_{k}$ and $\hat{N}_{k}$ in Figs. 2-4 and in the demonstrative movies [17]), streamwise-independent SFHs (perturbations with $k_{y}=0$ ) are not distinguished from streamwise-dependent SFHs in the self-sustenance process. Unlike in the 3D case, streamwiseindependent perturbations do not appear to be central participants in the dynamical processes. Instead, the dominant perturbations are shearwise independent (with $k_{x}=0$ ).

The basic equations of our research, Eqs. (6)-(9), were derived for 2D plane shear flows. However, as one can see from the Appendix, they also describe the local (small-scale) nonlinear dynamics of $2 \mathrm{D}$ rotating shear flows when the system is uniform in the direction $\hat{\mathbf{z}}$ of rotation. Such flows model local dynamical processes in unmagnetized astrophysical (galactic or protoplanet) disks, where selfsustenance of regular as well as stochastic/turbulent perturbations represents the key problem. The local approximation allows one to identify the $2 \mathrm{D}$ rotating case with the nonrotating case: within the limits of the local approach the mean flow vorticity is constant (equal to $A$ ) and the Rossby wave mode degenerates into a vortical perturbation. Thus, in the local approximation nonlinear processes are not affected by rotation, which vanishes from the dynamical equation.

Rotation changes the dynamical picture when the perturbation wavenumbers are nonzero in the $\hat{\mathbf{z}}$ direction $\left(k_{z} \neq 0\right)$ and the dynamics is three dimensional. Features of the dynamics in such a system, with rigid rotation, are outlined in detail in Chapter 4 of Ref. [18]. Even in the local approximation, perturbations in such a system exhibit wave behavior in the form of inertial waves. Consequently, the additional so-called selection rules for resonant triads restrict the possible nonlinear interactions. Wave turbulence differs essentially from hydrodynamic/eddy turbulence theory; it can be described by weak turbulence theory in terms of the evolution of slowly evolving envelopes that modulate highfrequency oscillations. This theory predicts a drain of energy onto the wave plane orthogonal to $\boldsymbol{\Omega}$. It establishes the tendency of even rigid rotating turbulence to become aniso- 
tropic due to spectral transfer on the horizontal wave plane (see [18]). The same tendency has also been observed in direct numerical and large-eddy simulations [19,20]. However, the anisotropy induced by rigid rotation does not affect the energetic balance of perturbations since there is no linear growth mechanism in the system. The situation will change in case of nonuniform rotation, where transient linear growth of perturbations is expected. Anisotropy in the plane orthogonal to $\hat{\mathbf{z}}$ should also appear, and consequently, the refined nonlinear processes should be more complex. A merger of the analyses in this work and in Ref. [18] would be a productive area for future research that could lead to a comprehensive understanding of the nature of refined nonlinearity.

In closing, we emphasize that NTR can introduce either a positive or negative feedback mechanism into turbulent dynamics. In the case of positive feedback, the nonlinearity repopulates growing perturbations and contributes to the self-sustenance of perturbations. Consequently, NTR naturally appears as a possible cornerstone of the bypass scenario of turbulence. In the case of negative feedback, the nonlinearity can suppress perturbations and may thus reduce anomalous transport phenomena. In fusion machines, a similar negative feedback scheme could perhaps be responsible for the L-H transition associated with poloidal shear flow.

\section{ACKNOWLEDGMENTS}

This work was supported by ISTC Grant No. G-1217 and by NSF Grant No. ATM-0638480. G.D.C. would like to acknowledge the hospitality of the Institute for Fusion Studies at the University of Texas at Austin.

\section{APPENDIX: 2D ROTATING SHEAR FLOW}

The governing ideal hydrodynamic equations of a twodimensional incompressible Keplerian disk flows in local Cartesian coordinates are

$$
\begin{gathered}
\frac{\partial \boldsymbol{U}}{\partial t}+\boldsymbol{U} \cdot \boldsymbol{\nabla} \boldsymbol{U}=-2 \Omega_{0} \hat{\mathbf{z}} \times \boldsymbol{U}+2 A \Omega_{0} x \hat{\mathbf{x}}-\nabla\left(\frac{P}{\rho_{0}}\right), \\
\boldsymbol{\nabla} \cdot \boldsymbol{U}=0,
\end{gathered}
$$

where a fiducial radius $r=r_{0}$ is introduced to replace the radial and azimuthal $(\theta)$ coordinates with local Cartesian coordinates,

$$
x \equiv r-r_{0}, \quad y \equiv r_{0}\left(\theta-\Omega_{0} t\right), \quad \frac{x}{r_{0}}, \frac{y}{r_{0}} \ll 1 .
$$

Here $\Omega_{0}$ is the local rotation angular velocity at $r=r_{0}$ and the local shear rate is defined by

$$
A \equiv-r_{0}\left[\frac{\partial \Omega(r)}{\partial r}\right]_{r=r_{0}}=\frac{3}{2} \Omega_{0} .
$$

The first term on the RHS of Eq. (A1) is the Coriolis force, and the second term accounts for the gravitational and centrifugal forces. The mean shear flow in the considered frame, which rotates with angular velocity $\Omega_{0}$, is $\boldsymbol{U}_{0}(0, A x)$. Splitting $\boldsymbol{U}$ into mean and perturbed (v) parts, Eqs. (A1) and (A2) reduce to the form

$$
\begin{aligned}
\left(\frac{\partial}{\partial t}+A x \frac{\partial}{\partial y}\right) \boldsymbol{v}+\boldsymbol{v} \cdot \boldsymbol{\nabla v} & =-2 \Omega_{0} v_{y} \hat{\mathbf{x}}+\left(2 \Omega_{0}-A\right) v_{x} \hat{\mathbf{y}} \\
& -\nabla\left(\frac{P}{\rho_{0}}\right), \\
\boldsymbol{\nabla} \cdot \boldsymbol{v} & =0 .
\end{aligned}
$$

On introducing the stream function $\psi$, defined by Eq. (2), and taking the curl of Eq. (A5), we finally obtain the inviscid form of our basic Eq. (1):

$$
\left(\frac{\partial}{\partial t}+A x \frac{\partial}{\partial y}\right) \Delta \psi+J(\psi, \Delta \psi)=0 .
$$

[1] S. C. Reddy and D. S. Henningson, J. Fluid Mech. 252, 209 (1993).

[2] L. Trefethen, A. Trefethen, S. Reddy, and T. Driscoll, Science 261, 578 (1993).

[3] W. O. Criminale, T. L. Jackson, and R. D. Joslin, Theory and Computation of Hydrodynamic Stability, Cambridge Monographs on Mechanics (Cambridge University Press, Cambridge, England, 2003).

[4] L. N. Trefethen and M. Embree, Spectra and Pseudospectra: The Behavior of Nonnormal Matrices and Operators (Princeton University Press, Princeton, NJ, 2005).

[5] P. J. Schmid, Annu. Rev. Fluid Mech. 39, 129 (2007).

[6] L. H. Gustavsson, J. Fluid Mech. 224, 241 (1991).

[7] B. F. Farrell and P. J. Ioannou, Phys. Fluids A 5, 1390 (1993).

[8] J. Baggett, T. Driscoll, and L. Trefethen, Phys. Fluids 7, 833 (1995).

[9] S. Grossmann, Rev. Mod. Phys. 72, 603 (2000).

[10] S. J. Chapman, J. Fluid Mech. 451, 35 (2002).
[11] B. Eckhardt, T. M. Schneider, B. Hof, and J. Westerweel, Annu. Rev. Fluid Mech. 39, 447 (2007).

[12] B. Eckhardt, H. Faisst, A. Schmiegel, and T. M. Schneider, Philos. Trans. R. Soc. London, Ser. A 366, 1297 (2008).

[13] C. E. Grosch and H. Salwen, J. Fluid Mech. 87, 33 (1978).

[14] H. Salwen and C. E. Grosch, J. Fluid Mech. 104, 445 (1981).

[15] S. Kida, J. Phys. Soc. Jpn. 50, 3517 (1981).

[16] J.-H. Kim, G. D. Chagelishvili, J. Perez, W. Horton, R. G. Chanishvili, J. G. Lominadze, and J. C. Bowman, Phys. Plasmas 13, 062304 (2006).

[17] http://pecos.ph.utexas.edu/ yegakjh

[18] P. Sagaut and C. Cambon, Homogeneous Turbulence Dynamics (Cambridge University Press, Cambridge, England, 2008).

[19] C. Cambon, N. N. Mansour, and F. S. Godeferd, J. Fluid Mech. 337, 303 (1997).

[20] Y. Morinishi, K. Nakabayashi, and S. Q. Ren, Phys. Fluids 13, 2912 (2001). 